\title{
The impact of the RASSF1C and PIWIL1 on DNA methylation: the identification of GMIP as a tumor suppressor
}

\author{
Yousef G. Amaar ${ }^{1}$ and Mark E. Reeves ${ }^{1,2}$ \\ ${ }^{1}$ Surgical Oncology Laboratory, Loma Linda VA Medical Center, Loma Linda, CA, USA \\ ${ }^{2}$ Loma Linda University Cancer Center, Loma Linda, CA, USA \\ Correspondence to: Yousef G. Amaar, email: yousef.amaar@va.gov \\ Keywords: lung cancer; RASSFIC; PIWIL1; DNA metylation \\ Received: May 11, $2020 \quad$ Accepted: October 17, $2020 \quad$ Published: November 10, 2020 \\ Copyright: ๑ 2020 Amaar and Reeves. This is an open access article distributed under the terms of the Creative Commons Attribution License \\ (CC BY 3.0), which permits unrestricted use, distribution, and reproduction in any medium, provided the original author and source are credited.
}

\section{ABSTRACT}

Introduction: Recently we have identified a novel RASSF1C-PIWIL1-piRNA pathway that promotes lung cancer cell progression and migration. PIWI-like proteins interact with piRNAs to form complexes that regulate gene expression at the transcriptional and translational levels. We have illustrated in previous work that RASSF1C modulates the expression of the PIWIL1-piRNA gene axis, suggesting the hypothesis that the RASSF1C-PIWI-piRNA pathway could potentially contribute to lung cancer stem cell development and progression, in part, through modulation of gene methylation of both oncogenic and tumor suppressor genes. Therefore, we tested this hypothesis using a non-small cell lung cancer (NSCLC) cell model to identify Candidate Differentially Methylated Regions (DMRs) modulated by the RASSF1C-PIWIL1-piRNA pathway.

Materials and Methods: We studied the impact of over-expressing RASSF1C and knocking down RASSF1C and PIWIL1 expression on global gene DNA methylation in the NSCLC cell line H1299 using the Reduced Representation Bisulfite Sequencing (RRBS) method.

Results: DMRs were identified by comparing DNA methylation profiles of experimental and control cells. Over-expression of RASSF1C and knocking down RASSF1C and PIWIL1 modulated DNA methylation of genomic regions; and statistically significant candidate genes residing DMR regions in lung cancer cells were identified, including oncogenes and tumor suppressors. One of the hypermethylated genes, Gem Interacting Protein (GMIP), displays tumor suppressor properties. GMIP expression attenuates lung cancer cell migration, and its over-expression is associated with longer survival of lung cancer patients.

Conclusions: The RASSF1C-PIWI-piRNA pathway modulates key oncogenes and tumor suppressor genes. GMIP is hypermethylated by this pathway and has tumor suppressor properties.

\section{INTRODUCTION}

Lung cancer is the most prevalent and most deadly cancer in the United States [1]. Thus, it is important to identify additional driver genes and their downstream pathways that can be targeted to effectively combat lung cancer. It is becoming evident that epigenomic and posttranscriptional regulation is critically important in human cancers. Indeed, epigenetically and post-transcriptionally regulated genes can be used as biomarkers for diagnosis, prognosis, molecular classification of tumors, targeted therapy, and predicting response to therapies. Hence, identification of new pathways and biomarkers for specific cancers is highly desirable for development of precision medicine tools. Our laboratory focuses on the oncogenic activities of the Ras Association Domain Family Member 1 (RASSF1) gene. RASSF 1 encodes two major isoforms, RASSF1A and RASSF1C, derived by alternative promoter selection and mRNA splicing [2,3]. RASSF1A is a tumor suppressor, whereas RASSF1C promotes cancer growth 
and migration [2-6]. Our previous studies show that a significant fraction of lung cancers are characterized by elevated RASSF1C or RASSF1C/RASSF1A ratios [6]. We have also demonstrated that RASSF1C stimulates in vitro cell cycle, proliferation, and migration of human lung cancer cells, size/number of tumor spheres produced by lung cancer stem cells (CSC), and in vivo tumor growth [4-9]. RASSF1C regulates expression of several genes/ proteins important in maintaining a CSC-like phenotype and oncogenesis [5]. In this regard, we have illustrated that RASSF1C modulates the expression of the PIWIL1piRNA gene axis $[5,9]$ suggesting the hypothesis that a RASSF1C-PIWI-piRNA pathway could potentially be a driver pathway that promotes lung cancer cell growth and progression. We have shown that RASSF1C induces expression of PIWIL1 and accumulation of $\beta$-catenin (both associated with stem cell self-renewal). In addition, we have shown that RASSF1C regulates expression of PIWI-interacting RNAs (piRNAs) associated with stem cell function. Further, small molecules that induce or attenuate RASSF1C expression (ERK inhibitor, CI-1040 and AMPK activator, Trichostatin A) have corresponding effects on PIWIL1 and piRNA gene expression [4, 5, 8, 9]. We have identified PIWIL1 as a potential regulator of Beta-catenin gene expression [8] and have identified specific piRNAs that appear to function as oncogenes (piR-34871 and piR-52200) and tumor suppressor (piR35127 and piR-46545) in normal and lung cancer cells [9]. Indeed, PIWIL1 over-expression has been shown to increase DNA methyltransferase 1 and 3a (DNMT1, DNMT3a) and methyl binding protein 2 (MBD2) [10, 11]. Also, PIWIL1 and its interacting piRNAs have been shown to inhibit apoptosis and promote DNA methylation of transposons and tumor suppressor genes [10, 11]. Further, PIWIL1 expression in lung tumors has been associated with shorter patient survival and has been suggested as an independent prognostic marker [12]. It has also been reported that somatic and malignant cells express unique sets of piRNA genes making it possible to distinguish between normal and malignant tissues in a cancer-type -specific manner [13]. Further, piRNAs have the potential to precisely define clinical features that include histological subgrouping, stage of disease, and survival [13]. Thus, gaining insights about the role PIWIL1-piRNA gene axis in human cancer growth and progression is very desirable.

As mentioned above, we have illustrated through published data that RASSF1C modulated the expression of PIWIL1-piRNA gene axis suggesting the hypothesis that RASSF1C-PIWI-piRNA pathway could potentially contribute to lung tumorigenesis, in part, through modulation of gene methylation (transcriptional regulation) and mRNA silencing (translational regulation) of both oncogenic and tumor suppressor genes. PIWIpiRNA-mediated transcriptional and posttranscriptional regulation remains largely unexplored in both normal and malignant human cells. To test our hypothesis, we conducted a global DNA methylation study to determine the impact of the RASSF1C-PIWIL1-pathway on DNA methylation in lung cancer cells. In this article, we report on Differentially Methylated Regions (DMRs) associated with specific candidate oncogenes and tumor suppressors which are impacted by modulating RASSF1C and PIWILI expression in lung cancer cells.

\section{RESULTS}

\section{RASSF1C-PIWIL1-piRNA pathway impact on global DNA methylation}

We studied the impact of over-expressing RASSF1C and knocking down RASSF1C and P1WIL1 expression on global gene DNA methylation. DNA from NCI-H1299 cells stably over-expressing RASSF1C, cells with RASSF1C knocked down, cells with PIWIL1 knocked down, or cells expressing sh-RNA vector control were used to perform global DNA methylation analyses using the RRBS method [14] to identify DMRs as candidates for new lung cancer biomarkers. Using selection criteria requiring a methylation fold-change of 1.5 or greater at a $p$-value $<0.05$, the RRBS methylation data analysis identified 99 candidate DMRs (out of over 80000 aligned RRBS reads, please see Supplementary Table 1) with methylation status in cells over-expressing RASSF1C that is opposite in cells with RASSF1 and PIWIL1 knockdowns. Our selection criteria ensured the most significant candidate DMRs targeted by the RASSF1C-PIWIL1-piRNA pathway are detected. Table 1 shows cytosine methylation analysis of NCI-H1299 cells expressing scrambled shRNA, overexpressing RASSF1C, expressing shRNA specific to RASSF1C, and expressing shRNA-specific to PIWIL1, respectively. In support of our hypothesis, potential oncogenes and tumor suppressors were among the 99 DMRs identified. Tables 2 and 3 list the top 4 oncogenes (A4GALT, CIB2, IRF4, and PSMA1) and tumor suppressor genes (GMIP, SPRED2, TBX5, and NKX2-1) associated with statistically significant DMRs, along with chromosomal position, number of CpGs mapped, methylation status, and methylation fold change. Interestingly, the $\mathrm{CpGs}$ identified fall within intragenic sequences (gene body) (Tables 2 and 3). It has been reported that intragenic DNA methylation in gene bodies is more frequent than in promoters and it may impact the transcriptional machinery efficiency and gene expression stability [15-17].

\section{Impact of identified oncogenes and tumor suppressor genes on lung cancer patient survival}

We have assessed the impact the top 4 oncogenes and tumor suppressor genes on survival of patients with lung adenocarcinoma using the Oncolnc database (http:// www.oncolnc.org) which links The Cancer Genome Atlas 
Table 1: Cytosine methylation analysis

\begin{tabular}{lccccc}
\hline Sample & Total C's analyzed & $\begin{array}{c}\text { Methylated C's in } \\
\text { CpG context }\end{array}$ & $\begin{array}{c}\text { \% methylation } \\
\text { (CpG context) }\end{array}$ & $\begin{array}{c}\text { \% methylation } \\
\text { (CHG context) }\end{array}$ & $\begin{array}{c}\text { \% methylation } \\
\text { (CHH context) }\end{array}$ \\
\hline Control & 451291731 & 56050505 & $59.8 \%$ & $0.8 \%$ & $0.6 \%$ \\
RASSF1C & 784227388 & 96950568 & $60.0 \%$ & $0.8 \%$ & $0.7 \%$ \\
RASSF1C-KD & 788105404 & 97441012 & $60.0 \%$ & $1.0 \%$ & $0.8 \%$ \\
PIWIL1-KD & 620576739 & 67623954 & $50.4 \%$ & $0.8 \%$ & $0.7 \%$ \\
\hline
\end{tabular}

Control: H1299 stably over-expression scrambled shRNA; RASSF1C: H1299 stably over-expressing RASSF1C; RASSF1C-KD: (C) H1299 stably overexpressing RASSF1C shRNA; PIWIL1-KD: NCI-H1299 stably over-expressing PIWIL1 shRNA.

survival data to the expression of mRNAs, miRNAs, and lncRNAs [18]. Kaplan-Meier analyses show that high expression of the oncogenes A4GALT, CIB2, and PSMA1 negatively correlates with patient survival (Figure 1). Kaplan-Meier analyses of the top four tumor suppressor genes show that high expression of both GMIP and NKX2-1 is associated with significantly longer patient survival. High expression of TBX5 and SPRED2 is not associated with significantly higher patient survival, although TBX5 expression is associated with a trend towards higher survival (Figure 2). TBX5 [19], SPRED2 [20], and NKX2-1 [21] have been shown to exhibit tumor suppressor functions in lung cancer, while GMIP function has yet to be determined in cancer cells.

\section{GMIP mRNA expression is in lung tumor tissue}

We assessed GMIP mRNA expression in lung cancer cells overexpressing RASSF1C and found that GMIP mRNA is down-regulated in cells overexpressing RASSF1C compared to cells overexpressing the control vector backbone (Figure 3). GMIP mRNA expression was up-regulated in cell with RASSF1C and PIWIL1 gene knock downs (Figure 3). We also assessed GMIP mRNA expression in human lung cancers and matched normal tissues and found that GMIP mRNA expression is down-regulated in $56 \%$ of lung cancer samples (Figure 4, $n=23$ ). Thus, GMIP could potentially be a novel lung cancer tumor suppressor.

\section{GMIP attenuates lung cancer cell migration}

Based on our Kaplan-Meier and mRNA expression analyses identifying GMIP as a potential tumor suppressor (Figure 2), we assessed the impact of overexpressing GMIP on lung cancer cells proliferation and migration. While GMIP overexpression did not affect cell proliferation (data not shown), GMIP overexpression in the lung cancer cell lines A549 and NCI-H1299 resulted in a significant decrease in cell migration (Figures 5 and 6).

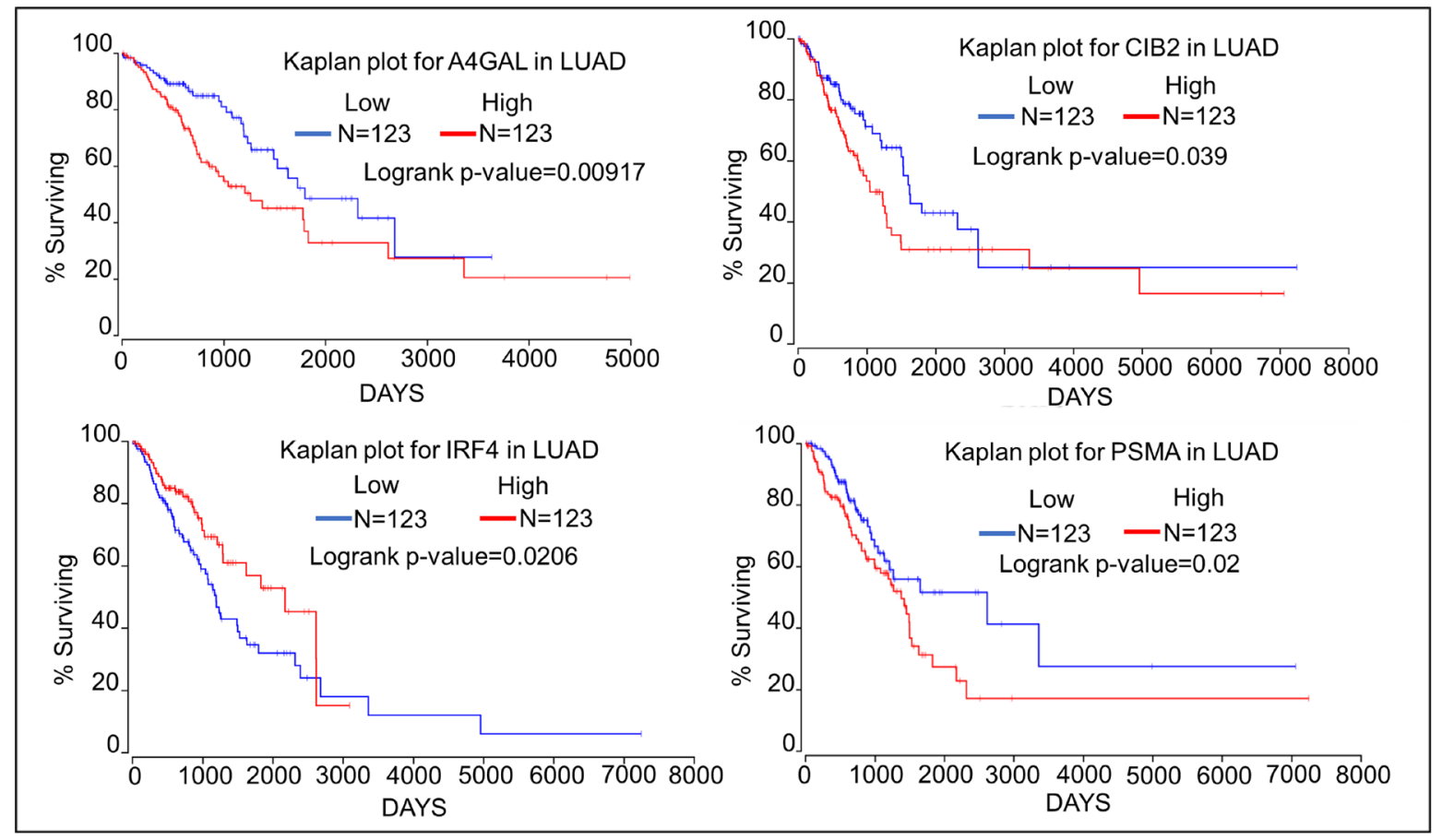

Figure 1: Kaplan-Meier plots showing the relationship of oncogene expression to lung cancer patient survival using data from TCGA. We compared the top 25 percentiles of High $(N=123)$ and low $(N=123)$ expressers of top four oncogene associated with DMRs. 


\section{GMIP gene contains potential piRNA binding sites}

GMIP has been identified as one of 3781 mRNAs predicted to be regulated by the PIWIL1-piRNA complex in mouse germ cells [22]. Since down-regulation of RASSF1C or PIWIL1 expression appears to decrease the methylation of GMIP, we hypothesized that the PIWIL1-piRNA complex could be involved in regulating GMIP gene expression via DNA methylation and/or gene silencing mechanism (s). Therefore, we scanned GMIP gene sequence for piRNA binding sites using tools available at the piRNA bank (http://pirnabank.ibab. ac.in/) [23]. This search showed that the GMIP gene contains 13 potential piRNA binding sites at the $3^{\prime}$ end. (Table 4). Taken together, these novel findings suggest that GMIP may be negatively regulated by the RASSF1CPIWIL1-piRNA pathway both at the transcriptional and translational levels.

\section{DISCUSSION}

In previous work, we have shown that the Ras Association Domain Family 1 Protein, isoform C (RASSF1C) promotes lung cancer cell proliferation, migration, drug-resistance, and attenuates apoptosis [4-9].
We also have shown that RASSF1C regulates the expression of the PIWIL1-piRNA gene axis, which is involved in promoting stem cell renewal $[5,8]$. We showed that overexpression of RASSF1C in LCSCs enhances the production and size of tumor spheres and implicated PIWIL1 as a potential mediator [8]. In addition, we have recently shown that RASSF1C appears to promote lung cell Epithelial to Mesenchymal Transition (EMT), in part, through regulation of miR-33a [27]. This, in turn, is a potential mechanism through which RASSF1C-PIWIL1-piRNA pathway may promote lung cancer cell metastasis. It has been reported that PIWIL1 promotes lung cancer cell proliferation, migration, and invasion; and PIWIL1 knockdown in LCSCs resulted in growth inhibition both in vitro and in vivo in a nude mouse model [24-26]. We should also note that PIWIL1 expression in lung tumor adenocarcinoma has been reported to be regulated by promoter DNA methylation and PIWIL expression has been correlated with the expression of gene signatures associated with stem cells [12, 24]. Elevated PIWIL1 expression in lung tumors is associated with poor patient prognosis and it has been suggested as an independent prognostic marker [12,24]. To increase our understanding of the impact of the RASSF1C-PIWIL1piRNA pathway on lung cancer, in this study we assessed the impact of the over-expressing RASSF1C and knocking down of RASSF1C and PIWIL1 genes on lung cancer cell

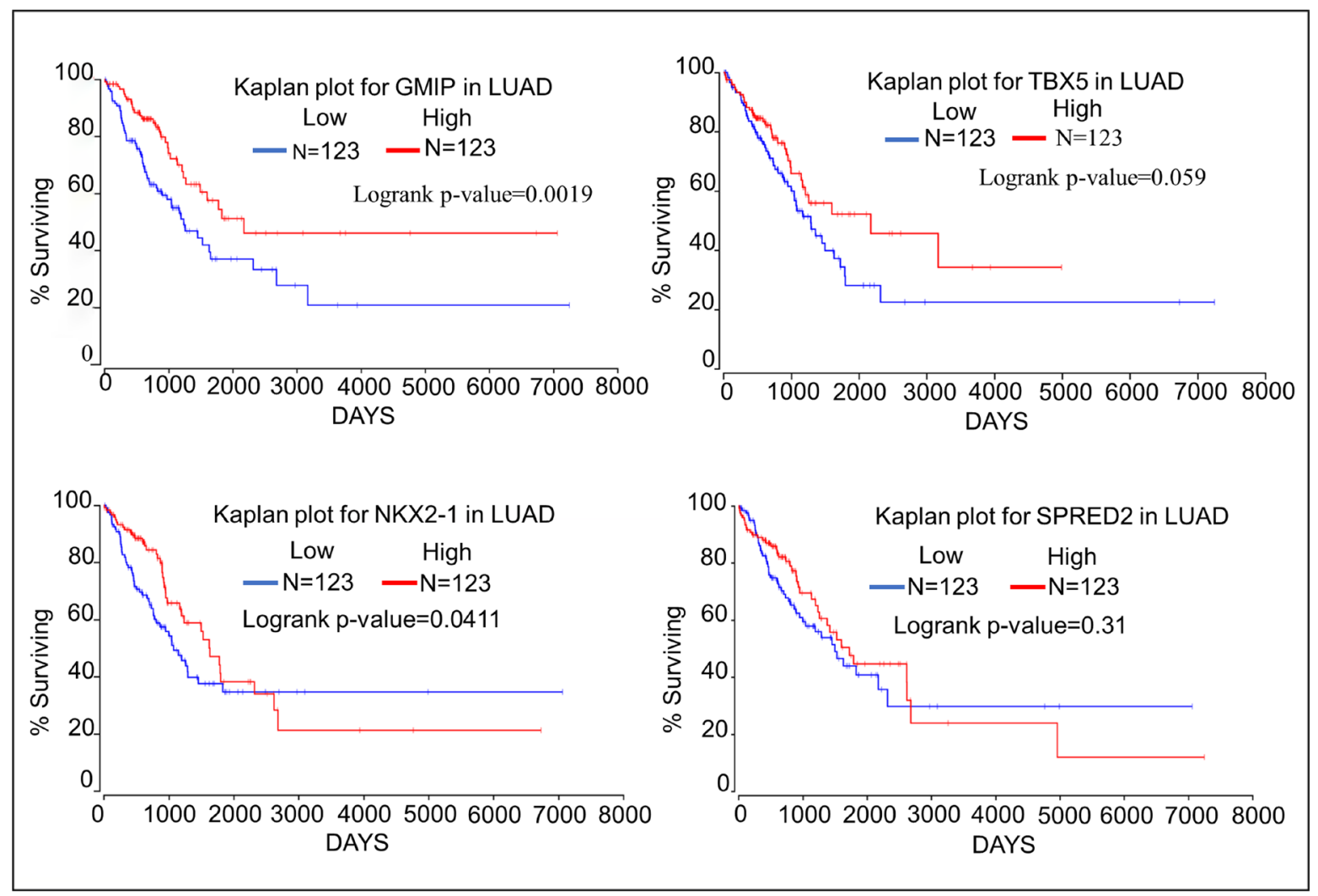

Figure 2: Kaplan-Meier plots showing the relationship of tumor suppressor gene expression to lung cancer patient survival using data from TCGA. We compared the top 25 percentiles of High $(N=123)$ and low $(N=123)$ expressers of top four oncogene associated with DMRs. 
Table 2: Top four oncogenes identified in DMRs regulated by the RASSF1C-PIWIL1-piRNA pathway

\begin{tabular}{|c|c|c|c|c|c|c|c|c|c|c|c|c|c|c|}
\hline Sample & Chr $^{*}$ & Start & end & Len & \#CpGs & $\begin{array}{l}+\&- \\
\text { Hits }\end{array}$ & $P$-value & Test & PropMeth & Gene & M-status & Fold_Diff & Strand & $\begin{array}{l}\text { Gene } \\
\text { Function }\end{array}$ \\
\hline RASSF1C & 11 & 14665235 & 14665441 & 207 & 18 & 6986 & $<0.001$ & $\mathrm{FE}$ & $\mathrm{R}=0.0095, \mathrm{~S}=0.0036$ & PSMA1 & Hypo-M & 2.6 & $3^{\prime}$ & Oncogene \\
\hline $1 \mathrm{C}-\mathrm{KD}$ & 11 & 14665235 & 14665441 & 207 & 18 & 8773 & 0.00 & $\mathrm{FE}$ & $\mathrm{R}=0.0095, \mathrm{~S}=0.0343$ & PSMA1 & Hyper-M & 3.6 & $3^{\prime}$ & Oncogene \\
\hline PIWIL1-KD & 11 & 14665235 & 14665441 & 207 & 18 & 4688 & 0.03 & FE & $\mathrm{R}=0.0095, \mathrm{~S}=0.0149$ & PSMA1 & Hyper-M & 1.6 & $3^{\prime}$ & Oncogene \\
\hline RASSF1C & 15 & 78423983 & 78424133 & 159 & 10 & 1224 & $<0.001$ & $\mathrm{FE}$ & $\mathrm{R}=0.0122, \mathrm{~S}=0.000$ & CIB2 & Нуро-М & 122 & $3^{\prime}$ & Oncogene \\
\hline $1 \mathrm{C}-\mathrm{KD}$ & 15 & 78423983 & 78423983 & 159 & 10 & 1067 & 0.013 & FE & $\mathrm{R}=0.0122, \mathrm{~S}=0.0325$ & CIB2 & Hyper-M & 2.7 & $3^{\prime}$ & Oncogene \\
\hline PIWIL1-KD & 15 & 78423983 & 78423983 & 159 & 10 & 1203 & $<0.001$ & $\mathrm{FE}$ & $\mathrm{R}=0.0122, \mathrm{~S}=0.0652$ & CIB2 & Hyper-M & 5.3 & $3^{\prime}$ & Oncogene \\
\hline RASSF1C & 6 & 391664 & 391864 & 201 & 13 & 1315 & $<0.001$ & $\mathrm{FE}$ & $\mathrm{R}=0.0631, \mathrm{~S}=0.0338$ & IRF4 & Нуро-М & 1.9 & $5^{\prime}$ & Oncogene \\
\hline $1 \mathrm{C}-\mathrm{KD}$ & 6 & 391664 & 391864 & 201 & 13 & 1354 & $<0.001$ & $\mathrm{FE}$ & $\mathrm{R}=0.0631, \mathrm{~S}=0.1739$ & IRF4 & Hyper-M & 2.8 & $5^{\prime}$ & Oncogene \\
\hline PIWIL1-KD & 6 & 391664 & 391864 & 201 & 13 & 1461 & 0.001 & $\mathrm{FE}$ & $\mathrm{R}=0.0631, \mathrm{~S}=0.1578$ & IRF4 & Hyper-M & 2.5 & $5^{\prime}$ & Oncogene \\
\hline RASSF1C & 22 & 43116675 & 43116842 & 168 & 20 & 823 & 0.038 & FE & $\mathrm{R}=0.0424, \mathrm{~S}=0.0213$ & A4GALT & Нуро-М & 2 & $3^{\prime}$-on_intron & Oncogene \\
\hline $1 \mathrm{C}-\mathrm{KD}$ & 22 & 43116675 & 43116842 & 168 & 20 & 572 & $<0.001$ & FE & $\mathrm{R}=0.0424, \mathrm{~S}=0.1371$ & A4GALT & Hyper-M & 3.2 & 3'-on_intron & Oncogene \\
\hline PIWIL1-KD & 22 & 43116675 & 43116842 & 168 & 20 & 862 & $<0.001$ & FE & $\mathrm{R}=0.0424, \mathrm{~S}=0.0797$ & A4GALT & Hyper-M & 1.9 & 3'-on_intron & Oncogene \\
\hline
\end{tabular}

Samples: RASSF1C = NCI-H1299 stably over-expressing RASSF1C; RASSF1C-KD = NCI-H1299 cells stably over-expression RASSF1C-shRNA (RASSF1C knocked down); PIWILI-KD = NCI-H1299 cells stably over-expressing PIWIL1-shRNA (PIWIL1 knocked down); R = NCI-H1299 over-expressinf scramble-shRNA (Control Reference); Legend: Chr": chromosome number; Start-end = starting-ending position; Len: length of $\mathrm{CpG}$ region; ${ }^{\#} \mathrm{CpGs}$ : number of $\mathrm{CpGs}$ in the region; +\&-hits: number of CpG hits per region; Test: FE: Fisher exact test; ProMeth: R: reference, S: sample; Gene: the name of the gene; M-Status: indicates hyper- or hypo- methylation. Bismark was used for the alignment and DMAP was used for the methylation differential analysis.

gene methylation. This identified several interesting gene targets including oncogenes (hypomethylated, Table 2) and tumor suppressors (Hypermethylated, Table 3) that are potentially regulated by this pathway. Using selection criteria requiring a methylation fold-change of 1.5 or greater at a $p$-value of $<0.05$, the RRBS methylation data analysis identified 99 candidate DMRs (out of over 80000 aligned RRBS reads) with a methylation status in cells overexpressing RASSF1C that is opposite that of cells with RASSF1 and PIWIL1 knocked down. Our selection criteria ensured that the most significant candidate DMRs targeted by the RASSF1C-PIWIL1-piRNA pathway are detected.

A4GALT, PSMA1, and CIB2 are all among the potential oncogenes identified, since high expression of their mRNAs is significantly associated with lower lung cancer patient survival (Figure 2). Interestingly, knockdown of PSMA1 has been shown to decrease radioresistance of NSCLC cells [28]. Conversely, CIB2 has been shown to attenuate oncogenic signaling in ovarian cancer, and low CIB2 expression is associated with poorer survival of ovarian cancer patients [29]. Thus, whether CIB2 acts as an oncogene in lung cancer remains to be determined. In contrast to A4GALT, CIB2, and PSMA1, high expression of IRF4 is associated with higher lung cancer patient survival. This appears to be inconsistent with published findings that IRF4 acts as an oncogene in NSCLC cell lines [30]. Like CIB2, IRF4 function in lung cancer remains to be clearly determined. Based on our findings identifying A4GAL as a novel candidate oncogene with potential biomarker value for lung cancer.
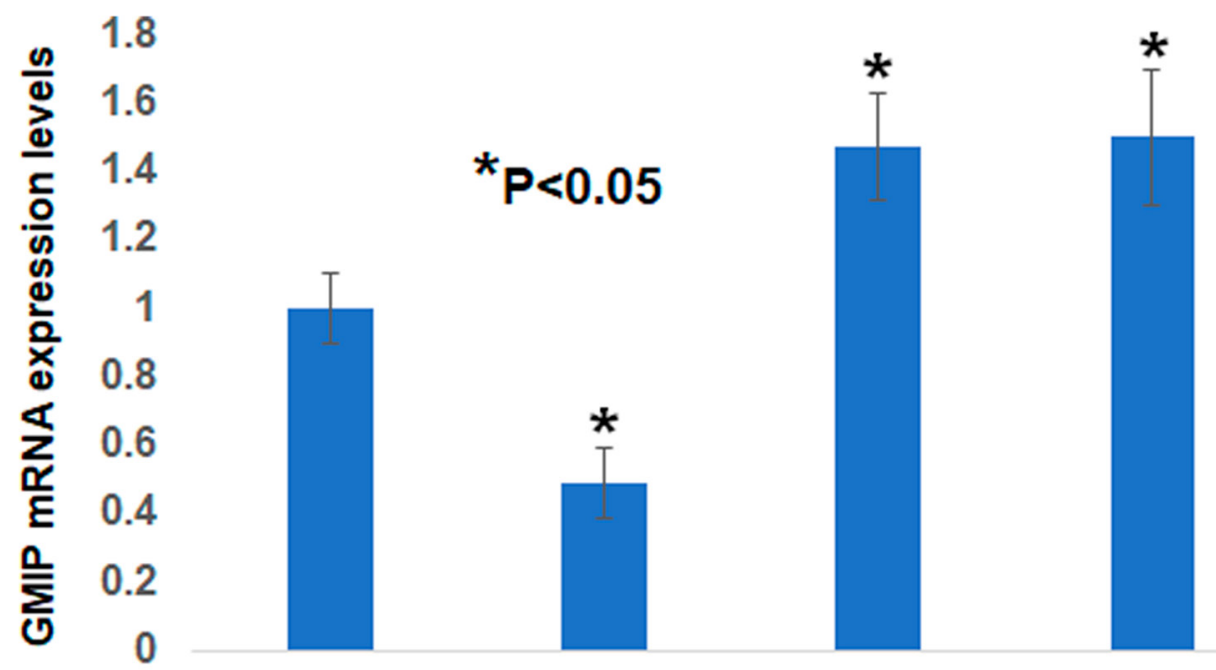

\section{Control RASSF1C RASSF1C-KD PIWIL1-KD}

Figure 3: Validation of GMIP gene expression by RT-PCR in NCI-H1299 lung cancer cells over-expressing vector backbone (H1299-BB) over-expressing RASSF1C (H1299-1C), H1299 cells with RASSF1C knockdown (RASSF1CKD), and PIWIL1-knockdown (PIWIL1-KD). RT-PCR were run in triplicates using the $2^{-\Delta \Delta C T}$ method with a $p$-value $<0.05$. 
Table 3: Top four tumor suppressors (TS) identified in DMRs regulated by the RASSF1C-PIWIL1piRNA pathway

\begin{tabular}{|c|c|c|c|c|c|c|c|c|c|c|c|c|c|c|}
\hline Sample & Chr\# & Start & end & Len & ${ }^{\#}$ CpGs & $\begin{array}{l}+\&- \\
\text { Hits }\end{array}$ & $P$-value & Test & PropMeth & Gene & M-status & $\begin{array}{l}\text { Fold } \\
\text { Diff }\end{array}$ & Strand & $\begin{array}{c}\text { Gene } \\
\text { Function }\end{array}$ \\
\hline RASSF1C & 19 & 19753727 & 19753881 & 155 & 5 & 193 & $<0.001$ & $\mathrm{FE}$ & $\mathrm{R}=0.1731, \mathrm{~S}=0.4382$ & Gmip & Hyper-M & 2.5 & 3'-on_intron & TS \\
\hline $1 \mathrm{C}-\mathrm{KD}$ & 19 & 19753727 & 19753881 & 155 & 5 & 140 & $<0.001$ & $\mathrm{FE}$ & $\mathrm{R}=0.1731, \mathrm{~S}=0.000$ & Gmip & Нуро-М & 1731 & $3^{\prime}$-on_intron & TS \\
\hline PIWIL1-KD & 19 & 19753727 & 19753881 & 155 & 5 & 191 & 0.01 & $\mathrm{FE}$ & $\mathrm{R}=0.1731, \mathrm{~S}=0.069$ & Gmip & Нуро-М & 2.5 & 3'-on_intron & TS \\
\hline RASSF1C & 2 & 65662753 & 65662944 & 192 & 9 & 1150 & 0.001 & $\mathrm{FE}$ & $\mathrm{R}=0.0537, \mathrm{~S}=0.1030$ & SPRED2 & Hyper-M & 1.9 & $3^{\prime}$ & TS \\
\hline $1 \mathrm{C}-\mathrm{KD}$ & 2 & 65662753 & 65662944 & 192 & 9 & 1026 & $<0.001$ & $\mathrm{FE}$ & $\mathrm{R}=0.0537, \mathrm{~S}=0.0238$ & SPRED2 & Нуро-М & 2.3 & $3^{\prime}$ & TS \\
\hline PIWIL1-KD & 2 & 65662753 & 65662944 & 192 & 9 & 914 & 0.046 & $\mathrm{FE}$ & $\mathrm{R}=0.0537, \mathrm{~S}=0.0399$ & SPRED2 & Нуро-М & 1.6 & $3^{\prime}$ & TS \\
\hline RASSF1C & 12 & 114886708 & 114886856 & 149 & 7 & 147 & 0.038 & $\mathrm{FE}$ & $\mathrm{R}=0.0631, \mathrm{~S}=0.0338$ & TBX5 & Hyper-M & 1.7 & $3^{\prime}$ & TS \\
\hline $1 \mathrm{C}-\mathrm{KD}$ & 12 & 114886708 & 114886856 & 149 & 7 & 83 & 0.02 & $\mathrm{FE}$ & $\mathrm{R}=0.0631, \mathrm{~S}=0.1739$ & TBX5 & Нуро-М & 1803 & $3^{\prime}$ & TS \\
\hline PIWIL1-KD & 12 & 114886708 & 114886856 & 149 & 7 & 133 & $<0.001$ & $\mathrm{FE}$ & $\mathrm{R}=0.0631, \mathrm{~S}=0.1578$ & TBX5 & Нуро-М & 6.5 & $3^{\prime}$ & TS \\
\hline RASSF1C & 14 & 36989325 & 36989495 & 171 & 13 & 749 & 0.003 & $\mathrm{FE}$ & $\mathrm{R}=0.0424, \mathrm{~S}=0.0213$ & NKX2-1 & Hyper-M & 1.7 & 3'-exon_intron & TS \\
\hline $1 \mathrm{C}-\mathrm{KD}$ & 14 & 36989325 & 36989495 & 171 & 13 & 1099 & $<0.001$ & FE & $\mathrm{R}=0.0424, \mathrm{~S}=0.1371$ & NKX2-1 & Нуро-М & 8.5 & 3'-exon_intron & TS \\
\hline PIWIL1-KD & 14 & 36989325 & 36989495 & 171 & 13 & 851 & $<0.001$ & $\mathrm{FE}$ & $\mathrm{R}=0.0424, \mathrm{~S}=0.0797$ & NKX2-1 & Нypo-M & 881 & 3'-exon intron & TS \\
\hline
\end{tabular}

Samples: RASSF1C $=$ NCI-H1299 stably over-expressing RASSF1C; RASSF1C-KD = NCI-H1299 cells stably over-expression RASSF1C-shRNA (RASSF1C knocked down); PIWILI-KD = NCI-H1299 cells stably over-expressing PIWIL1-shRNA (PIWIL1 knocked down); R = NCI-H1299 over-expressinf scrambleshRNA (Control Reference); Legend: Chr": chromosome number; Start-end = starting-ending position; Len: length of CpG region; ${ }^{~} \mathrm{CpGs}$ : number of CpGs in the region; +\&-hits: number of CpG hits per region; Test: FE: Fisher exact test; ProMeth: R: reference, S: sample; Gene: the name of the gene; M-Status: indicates hyper- or hypo- methylation. Bismark was used for the alignment and DMAP was used for the methylation differential analysis.

A4GALT mediation of glycosphingolipids is essential for the transition of cancer cells from mesenchymal to epithelial to initiate local tumor growth at the metastatic site [31]. As mentioned above RASSF1C promotes EMT of lung cancer cells [27], and others have shown the PIWIL1 induces EMT of endometrial cells [32] to promote metastasis. This raises the interesting hypothesis that the RASSF1C-PIWIL-piRNA pathway may promote reverse transition of metastatic cancer cells from mesenchymal to epithelial to permit local tumor growth at the new site of metastasis via demethylation and up-regulation of A4GALT gene expression. Further, the A4GALT enzyme is involved in globotriaosylceremede $(\mathrm{Gb} 3)$ biosynthesis. In addition, high levels of $\mathrm{Gb} 3$ have been shown to induce chemo-resistance in colon and lung cancer cell $[33,34]$. Thus, RASSF1C could promote lung cancer cell chemoresistance through modulation of A4GALT expression. We are currently exploring this hypothesis and determining the role of A4GALT in lung cancer and its relationship to the RASSF1C-PIWIL1-piRNA pathway.

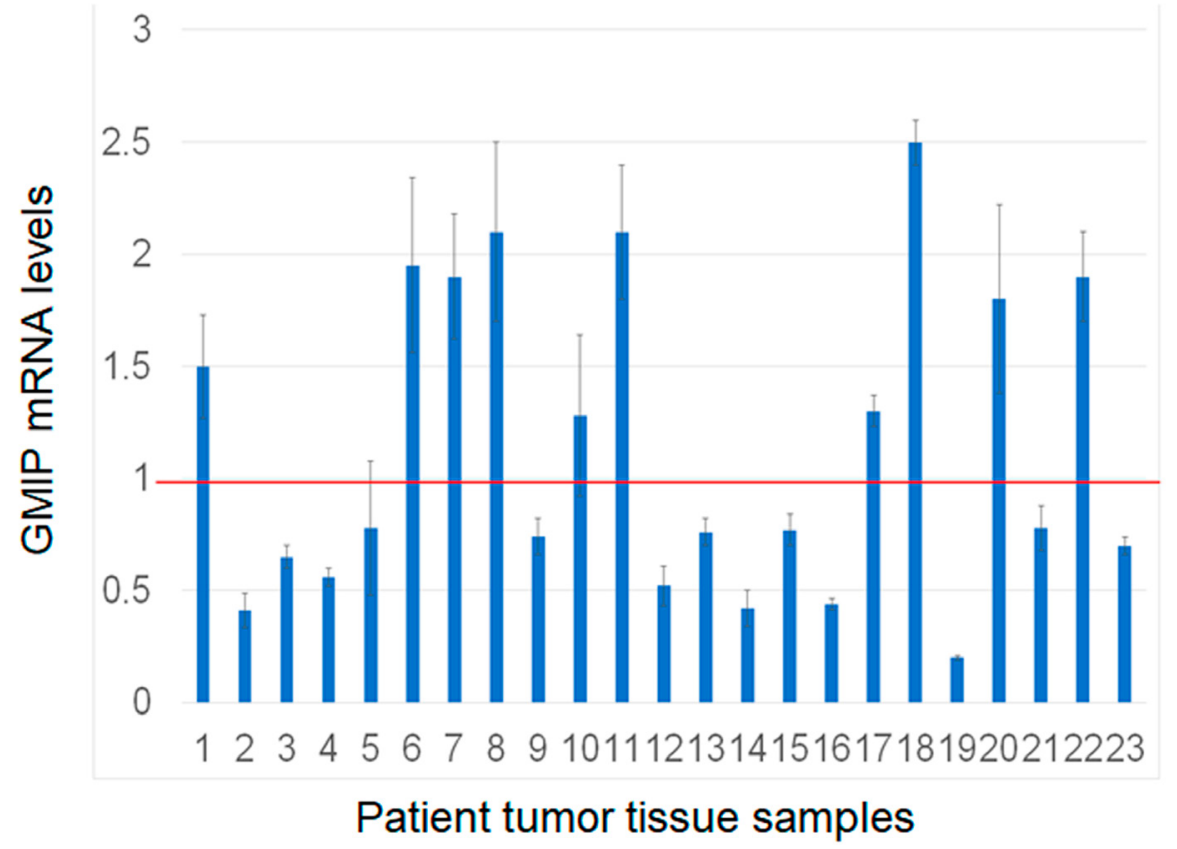

Figure 4: Assessment of GMIP gene expression by RT-PCR in human lung cancer and matched normal tissue samples $(\boldsymbol{n}=\mathbf{2 3})$. GMIP mRNA expression was lower in $13 / 23(56 \%)$ of tumor samples analyzed. The RT-PCR reactions were run in triplicates with $p$-value $<0.05$. 
Table 4: List of 13 piRNAs predicted within the 3'-region of the human GMIP gene

\begin{tabular}{lllll}
\hline Accession Id & piRNA Name & Length & Strand & Chromosomal position \\
\hline DQ578832 & hsa_piR_006465 & 30 & Plus & $19: 19607931-19607960$ \\
DQ582175 & hsa_piR_008983 & 28 & Plus & $19: 19603549-19603576$ \\
DQ585094 & hsa_piR_011187 & 30 & Minus & $19: 19612827-19612856$ \\
DQ585095 & hsa_piR_011188 & 30 & Minus & $19: 19612691-19612720$ \\
DQ590348 & hsa_piR_014879 & 31 & Minus & $19: 19612700-19612730$ \\
DQ590704 & hsa_piR_015150 & 29 & Plus & $19: 19603538-19603566$ \\
DQ590704 & hsa_piR_015150 & 29 & Plus & $19: 1960894-1960922$ \\
DQ590704 & hsa_piR_015150 & 29 & Minus & $19: 19612824-19612852$ \\
DQ592181 & hsa_piR_016271 & 29 & Minus & $19: 19603583-19603611$ \\
DQ592181 & hsa_piR_016271 & 29 & Plus & $19: 19613094-19613122$ \\
DQ593109 & hsa_piR_016792 & 31 & Plus & $19: 1960451-1960481$ \\
DQ593109 & hsa_piR_016792 & 31 & Minus & $19: 1960886-1960916$ \\
DQ601914 & hsa_piR_023338 & 29 & Plus & $19: 19603675-19603703$ \\
\hline
\end{tabular}

The piRNAs were predicted using piRNA-bank search tools.

Kaplan-Meier analyses show that high expression of both GMIP and NKX2-1 is significantly associated with higher lung cancer patient survival. However, high expression of TBX5 and SPRED2 is not associated with significantly higher lung cancer patient survival, although higher TBX5 expression shows a trend towards higher survival (Figure 3 ). It has been previously suggested that TBX5, SPRED, and NKX2-1 may function as tumor suppressors in lung cancer [19-21], while nothing is known about the function of GMIP in any human
A
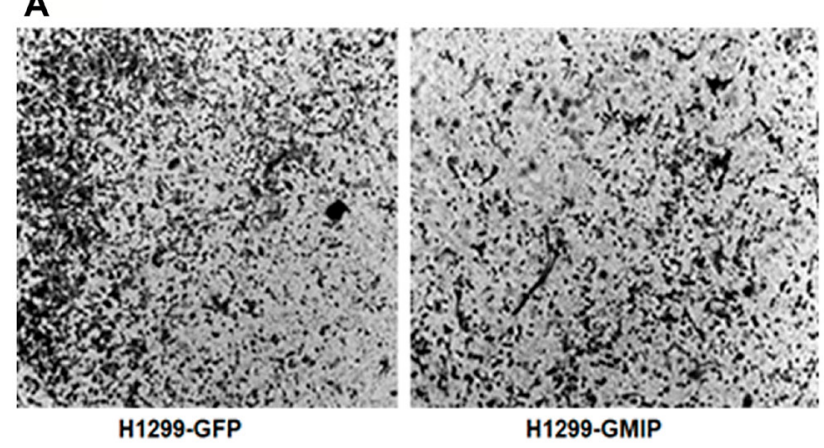

C

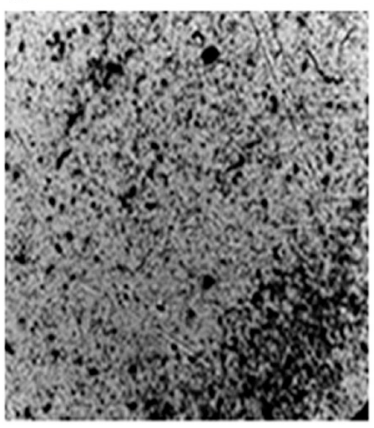

A549-GFP

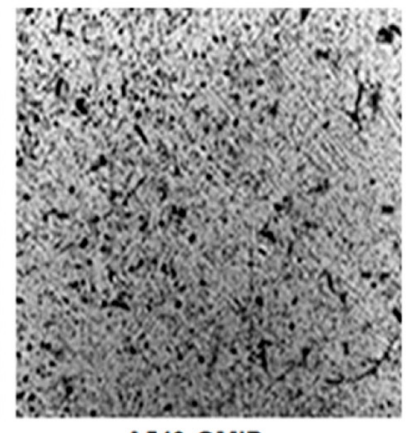

A549-GMIP
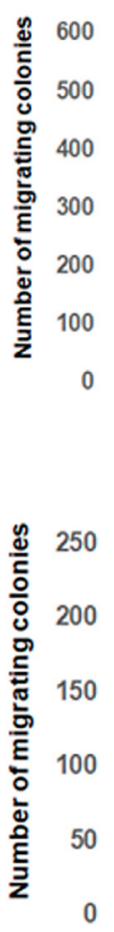

B

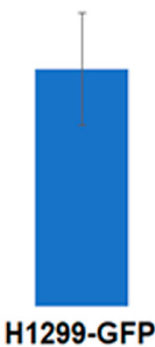

${ }^{\star} \mathrm{P}<0.05$

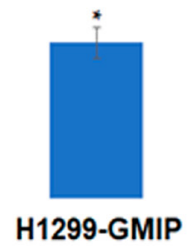

D

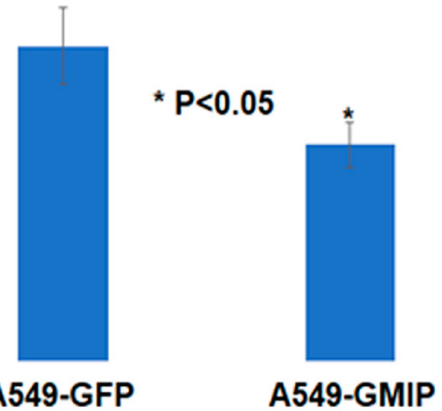

Figure 5: The BD BioCoatTM MatrigelTM Invasion Chamber was used to assess cell invasion/migration of H1299 and $\mathbf{A 5 4 9}$ cells transfected with GFP or GFP-GMIP plasmid. After $24 \mathrm{~h}$ incubation, the lower sides of the filters were fixed and stained H1299 (A) and A549 cells (C). Cells in four microscopic fields were counted and the average cell number was plotted. H1229 (B) and A549 (D) cells over-expressing GMIP showed a lower number of cell colonies invading the Matrigel chamber and migrating to the other side of the filter compared to H1299 and A549 transfected with GFP plasmid, $p<0.05$. The assay repeated three independent times. 
cancer. We should mention that GMIP is over-expressed in endometrial carcinoma and it has been suggested as a potential biomarker among several genes identified endometrial carcinoma [35]. However, the role of GMIP in endometrial carcinoma has not been determined yet. In this study we report for the first time that GMIP does display some characteristics of a novel tumor suppressor gene in lung cancer. This is supported by the methylation, cell migration, tumor mRNA expression, and KaplanMeier analyses from this study.

GMIP has previously been shown to inhibit mouse neuronal cell migration via inactivation of RhoA signaling [36]. In addition, RhoA activation has recently been shown to promote lung cancer EMT and metastasis [37]. In contrast to GMIP, PIWIL1 has been shown to promote mouse neuronal cell migration [38]. We have previously shown that RASSF1C promotes both lung and breast cancer cell migration [8]. Thus, our findings that knock-down of RASSF1C or PIWIL1 gene expression decreases GMIP Intron I intragenic methylation suggest that the RASSF1C-PIWIL1-piRNA pathway could, in part, promote cell migration by regulating GMIP gene expression. Consistent with a role for GMIP as an inhibitor of neuronal cell migration, we found that over-expression of GMIP in lung cancer cells appears to also attenuate lung cancer cell migration (Figure 5).

To gain more insight into how GMIP gene expression might be regulated by the RASSF1C-PIWIL1-piRNA pathway, we conducted a search using tools available at the piRNA bank website [23] to determine if the GMIP gene contains piRNA binding sites. Indeed, our search identified 13 potential piRNA binding sites in the $3^{\prime}$ end of GMIP gene (Table 4). This provides further support for the hypothesis that GMIP could be regulated by a PIWIL1piRNA complex in lung cancer cells. Also, in support of this hypothesis, a PIWIL1-CoIP study has identified GMIP as one of $3781 \mathrm{mRNAs}$ that are predicted to be regulated by the PIWIL1-piRNA complex in mouse germ cells [22]. Thus, GMIP gene expression regulation by the RASSF1CPIWIL1-piRNA pathway may occur perhaps both at the transcriptional and/or post-transcriptional levels.

In summary, our current data suggest that the RASSF1C-PIWIL1-piRNA pathway appears to play a role in modulating methylation of oncogenes and tumor suppressors to promote lung cancer cell growth and progression. In addition, they also suggest that A4GALT and GMIP may be new biomarkers for lung cancer that will be the focus of future investigations.
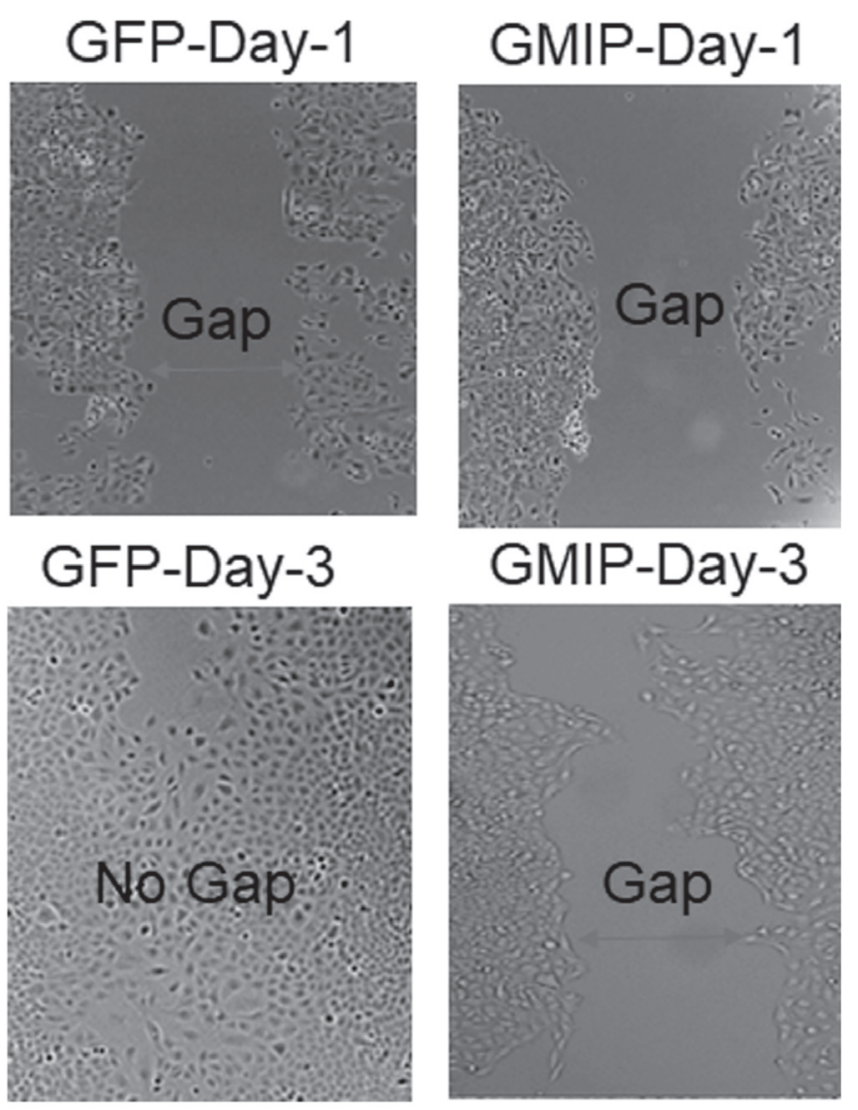

Figure 6: GMIP over-expression inhibits lung cancer cell migration. A549 cells were transiently transfected with either GFP plasmid (Control) or GFP-GMIP plasmid culture-inserts for wound healing assays crating a cell-free gap of $500 \mu \mathrm{m}$. Then cells were monitored for cap closure for four days post-transfection. The gap (wound) between A549 cells transfected with GFP was closed by Day-3 compared to A549 cells over-expressing GFP-GMIP which was not closed. 


\section{MATERIALS AND METHODS}

\section{Cell culture}

The human non-small cell lung cancer (NSCLC) cell line NCI-H1299 stably over-expressing RASSF1C (H1299-1C), cells with RASSF1C knocked down (cell stably over-expressing sh-RNA specific to RASSF1C), with PIWIL1 knocked down (Cells stably over-expressing sh-RNA specific to PIWIL1), or cells stably expressing sh-RNA vector control. Cells were grown in RPMI-1640 medium supplemented with $10 \%$ calf bovine serum as previously described [8].

\section{DNA methylation analysis}

We studied the impact of over-expressing and silencing RASSF1C and silencing of PIWIL1 genes on global gene DNA methylation in NSCLC cells (NCI-H1299). DNA from cells over-expressing RASSF1C, cells with RASSF1C knocked down, cells with PIWIL1 knocked down, or cells expressing sh-RNA vector control was isolated and one sample from each cell line was used for DNA methylation analyses using the Reduced Representation Bisulfite Sequencing (RRBS) method [10] at the Technology Center for Genomics and Bioinformatics (TCGB, UCLA, CA, USA). Bioinformatic analysis was conducted at the TCGB to identify DMRs. Bismark, a software package to map and determine the methylation state of BS-Seq reads, was used for the alignment and DMAP (differential methylation analysis package) [14] was used for the methylation differential analysis. DMAP includes Chi-square, Fisher's exact and analysis of variance (ANOVA) statistical tests, to identify methylation differences between different groups and conditions. DMAP also specifies information on genomic relationship including nearest gene, exon, introns and $\mathrm{CpG}$ features) for every DMR. Statistically significant candidate genes with hypo- and hyper-methylated C islands were identified. RRBS reduces the portion of the genome analyzed through MspI digestion and fragment size selection. Using RRBS, DNA sequencing is focused on well-defined $\mathrm{CpG}$ rich 'reduced representation' of the genome and it measures DNA methylation based on DNA sequence in each region [10].

\section{Kaplan-Meier analyses of potential oncogenes and tumor suppressors}

The top 4 putative oncogenes and tumor suppressor genes were identified based on differential hypo- and hypermethylation, respectively. The impact of these genes on survival of patients with lung adenocarcinoma was assessed using the Oncolnc database, with KaplanMeier survival analysis with log rank significance testing based on mRNA expression level. This database links The
Cancer Genome Atlas survival data to the expression of mRNAs, miRNAs, and lncRNAs. It presents over 400,00 analyses that includes Multivariate Cox regressions analysis [18].

\section{RT-PCR analysis}

Total RNA from human lung cancers and matched normal tissues was isolated and reverse transcriptase (RT)-PCR was performed using gene-specific primers as previously described (6). PCR was carried out using HotStart and Sybergreen master mixes (Qiagen, Valencia, CA, USA). The RT-PCR reactions were carried out in triplicate and the fold change was calculated using the $2^{-\Delta \Delta C T}$ method [39]. GMIP RT-PCR analysis was carried out using GMIP specific gene primers and Cyclophilin gene expression (internal control) was assessed using gene specific primers. GMIP-F: CTTGAACAGCTCCCCTCTGG and GMIP-R: CTGGAGTCCCTTGCCAGC.

\section{Cell migration assay}

NCI-H1299 cells were plated at $2 \times 10^{4}$ cells per chamber and were transfected the next day with GFP or GFP-GMIP plasmid. Cells were transfected with $0.2 \mu \mathrm{g}$ of plasmid DNA per cell culture insert and with $0.4 \mu \mathrm{g}$ plasmid DNA per Bowden chamber using Lipofectamine 2000. For the Bowden chamber assay, cells were processed 24-48 h post-transfection, and were fixed with methanol for two min, and stained with $1 \%$ Toludine blue for two min as previously described [8]. The stained Bowden chambers were examined under the bright field microscope and were photographed.

\section{Statistical analysis}

The $t$-test was used to calculate the significance of data.

\section{Author contributions}

Yousef Amaar designed and supervised the study, carried out data analysis and drafting of the manuscript. Mark Reeves participated in the design of the study, contributed to data analysis, and drafting of the manuscript.

\section{ACKNOWLEDGMENTS AND FUNDING}

This work was carried out at the Loma Linda VA Medical Center, Loma Linda, CA. It was supported by a seed grant from the Loma Linda University Cancer Center.

\section{CONFLICTS OF INTEREST}

Authors have no conflicts of interest to declare. 


\section{REFERENCES}

1. Siegel RL, Miller KD, Jemal A. Cancer statistics, 2020. CA Cancer J Clin. 2020; 70:7-30. https://doi.org/10.3322/ caac.21590. [PubMed]

2. Dammann R, Takahashi T, Pfeifer G. The CpG island of the novel tumor suppressor gene RASSF1A is intensely methylated in primary small cell lung carcinoma. Oncogene. 2001; 20:3563-3567. https://doi.org/10.1038/ sj.onc.1204469. [PubMed]

3. Burbee DG, Forgacs E, Zochbauer-Muller S, Shivakumar L, Fong K, Gao B, Randle D, Kondo M, Virmani A, Bader S, Sekido Y, Latif F, Milchgrub S, et al. Epigenetic inactivation of RASSF1A in lung and breast cancers and malignant phenotype suppression. J Natl Cancer Inst. 2001; 93:691699. https://doi.org/10.1093/jnci/93.9.691. [PubMed]

4. Amaar YG, Minera MG, Hatran LK, Strong DD, Mohan $\mathrm{S}$, Reeves ME. Ras association domain family $1 \mathrm{C}$ protein stimulates human lung cancer cell proliferation. Am J Physiol Lung Cell Mol Physiol. 2006; 291:L1185-1190. https://doi.org/10.1152/ajplung.00072.2006. [PubMed]

5. Reeves ME, Baldwin ML, Aragon RJ, Baldwin SW, Chen ST, Li X, Mohan S, Amaar YG. RASSF1C modulates the expression of a stem cell renewal gene, PIWIL1. BMC Res Notes. 2012; 5:239. https://doi.org/10.1186/1756-0500-5239. [PubMed]

6. Reeves ME. Firek M, Chen ST, Amaar Y. The RASSF1 Gene and the Opposing Effects of the RASSF1A and RASSF1C Isoforms on Cell Proliferation and Apoptosis. Mol Biol Int. 2013; 2013:145096. https://doi. org/10.1155/2013/145096. [PubMed]

7. Reeves ME, Aragon RJ, Alfakhori M, Chen ST, Lowen N, Mohan S, Amaar YG. Ras-Association Domain Family 1C protein Enhances Breast Tumor Growth In Vivo. Cancer Growth Metastasis. 2012; 5:27-33. https://doi.org/10.4137/ CGM.S9845.

8. Reeves ME, Firek M, Chen ST, Amaar YG. Evidence that RASSF1C stimulation of lung cancer cell proliferation depends on IGFBP-5 and PIWIL1 expression levels. PLoS One. 2014; 9:e101679. https://doi.org/10.1371/journal. pone.0101679. [PubMed]

9. Reeves ME, Firek M, Jliedi A, Amaar YG. Identification and characterization of RASSF1C piRNA target genes in lung cancer cells. Oncotarget. 2017; 8:34268-34282. https://doi.org/10.18632/oncotarget.15965. [PubMed]

10. Siddiqi S, Matushansky I. Piwis and piwi-interacting RNAs in the epigenetics of cancer. J Cell Biochem. 2012; 113:373-380. https://doi.org/10.1002/jcb.23363. [PubMed]

11. Liu J, Zhang S, Cheng B. Epigenetic roles of PIWIinteracting RNAs (piRNAs) in cancer metastasis. Oncol Rep. 2018; 40:2423-2434. https://doi.org/10.3892/ or.2018.6684. [PubMed]

12. Navarro A, Tejero R, Viñolas N, Cordeiro A, Marrades RM, Fuster D, Caritg O, Moises J, Muñoz C, Molins L,
Ramirez J, Monzo M. The significance of PIWI family expression in human lung embryogenesis and non-small cell lung cancer. Oncotarget. 2015; 6:31544-31556. https:// doi.org/10.18632/oncotarget.3003. [PubMed]

13. Martinez VD, Vucic EA, Thu KL, Hubaux R, Enfield KS, Pikor LA, Becker-Santos DD, Brown CJ, Lam S, Lam WL. Unique somatic and malignant expression patterns implicate PIWI-interacting RNAs in cancer-type specific biology. Sci Rep. 2015; 5:10423. https://doi.org/10.1038/srep10423. [PubMed]

14. Bock C, Tomazou EM, Brinkman AB, Müller F, Simmer F, Gu H, Jäger N, Gnirke A, Stunnenberg HG, Meissner A. Quantitative comparison of genome-wide DNA methylation mapping technologies. Nat Biotechnol. 2010; 28:11061114. https://doi.org/10.1038/nbt.1681. [PubMed]

15. Ehrlich M, Lacey M. DNA Methylation and differentiation: silencing, upregulation and modulation of gene expression. Epigenomics. 2013; 5:553-68. https://doi.org/10.2217/ epi.13.43. [PubMed]

16. Rauscher GH, Kresovich JK, Poulin M, Yan L, Macias V, Mahmoud AM, Al-Alem U, Kajdacsy-Balla A, Wiley EL, Tonetti D, Ehrlich M. Exploring DNA methylation changes in promoter, intragenic, and intergenic regions as early and late events in breast cancer formation. BMC Cancer. 2015; 15:816. https://doi.org/10.1186/s12885-015-1777-9. [PubMed]

17. Jeziorska DM. Murray RJS, De Gobbi M, Gaentzsch R, Garrick D, Ayyub H, Chen T, Li E, Telenius J, Lynch M, Graham B, Smith AJH, Lund JN, et al. DNA methylation of intragenic $\mathrm{CpG}$ islands depends on their transcriptional activity during differentiation and disease. Proc Natl Acad Sci U S A. 2017; 114:E7526-E7535. https://doi.org/10.1073/ pnas.1703087114. [PubMed]

18. Anaya J. OncoLnc: linking TCGA survival data to mRNAs, miRNAs, and lncRNAs. PeerJ Comput Sci. 2016; 2:e67. https://doi.org/10.7717/peerj-cs.67.

19. Ma R, Yang Y, Tu Q, Hu K. Overexpression of T-box Transcription Factor 5 (TBX5) Inhibits Proliferation and Invasion in Non-Small Cell Lung Carcinoma Cells. Oncol Res. 2017; 25:1495-1504. https://doi.org/10.3727/0965040 17X14883287513729. [PubMed]

20. Jiang K, Liu M. Lin G, Mao B, Cheng W, Liu H, Gal J, Zhu H, Yuan Z, Deng W, Liu Q, Gong P, Bi X, Meng S. Tumor suppressor Spred2 interaction with LC3 promotes autophagosome maturation and induces autophagydependent cell death. Oncotarget. 2016; 7:25652-25667. https://doi.org/10.18632/oncotarget.8357. [PubMed]

21. Caswell DR. Chuang CH, Ma RK, Winters IP, Snyder EL, Winslow MM. Tumor Suppressor Activity of Selenbp1, a Direct Nkx2-1 Target, in Lung Adenocarcinoma. Mol Cancer Res. 2018; 16:1737-1749. https://doi. org/10.1158/1541-7786.MCR-18-0392. [PubMed]

22. Yuan J, Zhang P, Cui Y, Wang J, Skogerbø G, Huang DW, Chen R, He S. Computational identification of piRNA 
targets on mouse mRNAs. Bioinformatics. 2016; 32:1170 1177. https://doi.org/10.1093/bioinformatics/btv729. [PubMed]

23. Sai Lakshmi S, Agrawal S. piRNABank: a web resource on classified and clustered Piwi-interacting RNAs. Nucleic Acids Res. 2008; 36:D173-7. https://doi.org/10.1093/nar/ gkm696. [PubMed]

24. Xie K, Zhang K, Kong J, Wang C, Gu Y, Liang C, Jiang T, Qin N, Liu J, Guo X, Huo R, Liu M, Ma H, et al. Cancertestis gene PIWIL1 promotes cell proliferation, migration, and invasion in lung adenocarcinoma. Cancer Med. 2018; 7:157-166. https://doi.org/10.1002/cam4.1248. [PubMed]

25. Liang D, Dong M, Hu LJ, Fang ZH, Xu X, Shi EH, Yang YJ. Hiwi knockdown inhibits the growth of lung cancer in nude mice. Asian Pac J Cancer Prev. 2013; 14:1067-1072. https://doi.org/10.7314/APJCP.2013.14.2.1067. [PubMed]

26. Chen X, Su Y, Fingleton B, Acuff H, Matrisian LM, Zent R, Pozzi A. An orthotopic model of lung cancer to analyze primary and metastatic NSCLC growth in integrin alpha1null mice. Clin Exp Metastasis. 2005; 22:185-193. https:// doi.org/10.1007/s10585-005-7453-8. [PubMed]

27. Amaar YG, Reeves ME. RASSF1C regulates miR-33a and EMT marker gene expression in lung cancer cells. Oncotarget. 2019; 10:123-132. https://doi.org/10.18632/ oncotarget.26498. [PubMed]

28. Cron KR, Zhu K, Kushwaha DS, Hsieh G, Merzon D, Rameseder J, Chen CC, D'Andrea AD, Kozono D. Proteasome inhibitors block DNA repair and radiosensitize non-small cell lung cancer. PLoS One. 2013; 8:e73710. [PubMed]

29. Zhu W, Jarman KE, Lokman NA, Neubauer HA, Davies LT, Gliddon BL, Taing H, Moretti PAB, Oehler MK, Pitman MR, Pitson SM. CIB2 Negatively Regulates Oncogenic Signaling in Ovarian Cancer via Sphingosine Kinase 1. Cancer Res. 2017; 77:4823-4834. https://doi. org/10.1158/0008-5472.CAN-17-0025. [PubMed]

30. Qian Y, Du Z, Xing Y, Zhou T, Chen T, Shi M. Interferon regulatory factor 4 (IRF4) is overexpressed in human nonsmall cell lung cancer (NSCLC) and activates the Notch signaling pathway. Mol Med Rep. 2017; 16:6034-6040. https://doi.org/10.3892/mmr.2017.7319. [PubMed]

31. Jacob F, Alam S, Konantz M, Liang CY, Kohler RS, Everest-Dass AV, Huang YL, Rimmer N, Fedier A, Schötzau A, Lopez MN, Packer NH, Lengerke C, HeinzelmannSchwarz V. Transition of Mesenchymal and Epithelial Cancer Cells Depends on a1-4 GalactosyltransferaseMediated Glycosphingolipids. Cancer Res. 2018; 78:29522965. https://doi.org/10.1158/0008-5472.CAN-17-2223. [PubMed]
32. Chen Z, Che Q, He X, Wang F, Wang H, Zhu M, Sun J, Wan $\mathrm{X}$. Stem cell protein Piwill endowed endometrial cancer cells with stem-like properties via inducing epithelialmesenchymal transition. BMC Cancer. 2015; 15:811. https://doi.org/10.1186/s12885-015-1794-8. [PubMed]

33. Kovbasnjuk O. Mourtazina R, Baibakov B, Wang T, Elowsky C, Choti MA, Kane A, Donowitz M. The glycosphingolipid globotriaosylceramide in the metastatic transformation of colon cancer. Proc Natl Acad Sci U S A. 2005; 102:19087-19092. https://doi.org/10.1073/ pnas.0506474102. [ubMed]

34. Tyler A. Johansson A, Karlsson T, Gudey SK, Brännström T, Grankvist K, Behnam-Motlagh P. Targeting glucosylceramide synthase induction of cell surface globotriaosylceramide (Gb3) in acquired cisplatin-resistance of lung cancer and malignant pleural mesothelioma cells. Exp Cell Res. 2015; 336:23-32. https://doi.org/10.1016/j. yexcr.2015.05.012. [PubMed]

35. Colas E, Perez C, Cabrera S, Pedrola N, Monge M, Castellvi J, Eyzaguirre F, Gregorio J, Ruiz A, Llaurado M, Rigau M, Garcia M, Ertekin T, et al. Molecular markers of endometrial carcinoma detected in uterine aspirates. Int J Cancer. 2011; 129:2435-2444. https://doi.org/10.1002/ ijc.25901. [PubMed]

36. Ota H, Hikita T, Sawada M, Nishioka T, Matsumoto M, Komura M, Ohno A, Kamiya Y, Miyamoto T, Asai N, Enomoto A, Takahashi M, Kaibuchi K, et al. Speed control for neuronal migration in the postnatal brain by GMIPmediated local inactivation of RhoA. Nat Commun. 2014; 30:4532. https://doi.org/10.1038/ncomms5532. [PubMed]

37. Salvi A, Thanabalu T. WIP promotes in-vitro invasion ability, anchorage independent growth and EMT progression of A549 lung adenocarcinoma cells by regulating RhoA levels. Biochem Biophys Res Commun. 2017; 482:13531359. https://doi.org/10.1016/j.bbrc.2016.12.040. [PubMed]

38. Zhao PP, Yao MJ, Chang SY, Gou LT, Liu MF, Qiu ZL, Yuan $\mathrm{XB}$. Novel function of PIWIL1 in neuronal polarization and migration via regulation of microtubule-associated proteins. Mol Brain. 2015; 8:39. https://doi.org/10.1186/s13041-0150131-0. [PubMed]

39. Livak KJ, Schmittgen TD. Analysis of relative gene expression data using real-time quantitative PCR and the 2(-Delta Delta C(T)) Method. Methods. 2001; 25:402-408. https://doi.org/10.1006/meth.2001.1262. [PubMed] 